\title{
Robust Adaptive Reactive Power Control for Doubly Fed Induction Generator
}

\author{
Huabin Wen, ${ }^{1}$ Yu Zeng, ${ }^{2}$ Lei Wang, ${ }^{3}$ Feng Yang, ${ }^{4}$ and Y. D. Song ${ }^{3}$ \\ ${ }^{1}$ School of Electrical Engineering, Beijing Jiaotong University, Beijing 100044, China \\ ${ }^{2}$ School of Energy Science and Engineering, UESTC, Chengdu 611731, China \\ ${ }^{3}$ School of Automation, Chongqing University, Chongqing 400044, China \\ ${ }^{4}$ School of Automation Engineering, UESTC, Chengdu 611731, China
}

Correspondence should be addressed to Y. D. Song; ydsong@cqu.edu.cn

Received 3 February 2014; Accepted 19 March 2014; Published 20 May 2014

Academic Editor: Peng Shi

Copyright (C) 2014 Huabin Wen et al. This is an open access article distributed under the Creative Commons Attribution License, which permits unrestricted use, distribution, and reproduction in any medium, provided the original work is properly cited.

\begin{abstract}
The problem of reactive power control for mains-side inverter (MSI) in doubly fed induction generator (DFIG) is studied in this paper. To accommodate the modelling nonlinearities and inherent uncertainties, a novel robust adaptive control algorithm for MSI is proposed by utilizing Lyapunov theory that ensures asymptotic stability of the system under unpredictable external disturbances and significant parametric uncertainties. The distinguishing benefit of the aforementioned scheme consists in its capabilities to maintain satisfactory performance under varying operation conditions without the need for manually redesigning or reprogramming the control gains in contrast to the commonly used PI/PID control. Simulations are also built to confirm the correctness and benefits of the control scheme.
\end{abstract}

\section{Introduction}

Doubly fed induction generator (DFIG) enjoys more noticeable advantages compared with other kinds of wind generators [1]. For example, by keeping the rotor current frequency at a constant level, DFIG can produce nearly constant power from the stator, and by keeping an optimal tip-speed ratio, DFIG is able to capture the maximum wind power at different wind speeds [2]. A wind power generation system equipped with DFIG requires a converter with only one-third of the power rating, leading to a less expensive system with reduced power loss [3]. DFIG can also control reactive power separately from active power with a reasonable adoption of orientation frame [4]. Especially, DFIG can stabilize the power network voltage by providing some controllable reactive power, thus improving power factor or voltage characteristics [5].

Researches on the blackout in Canada and America in 2003 indicated that if reactive power was provided in time the cascaded outages of several power system devices might have been avoided. Reactive power is closely related to voltage level and power factor (pf) and terminal voltage. To prevent power network instability problem, some power companies have proposed several standards which must be strictly met when the wind generators connect to the system [6]. Therefore, reactive power control in DFIG for wind turbines has become a research topic of theoretical and practical importance that has attracted considerable attention during the past decade, leading to a number of technical results on reactive power control of DFIG in wind turbines. Brekken and Mohan [7] deal with the harmonic component on the frame with a low bandwidth filter. A PI and a state space based controller for reactive power are studied by Machmoum et al. [8]. The limitation of generation capability on both converters of DFIG is analysed in Engelhardt et al. [9]. Slootweg et al. [10] study the voltage control scheme by reactive power compensation on the RSI, without considering reactive power generation ability of grid-side inverter (MSI). In Tapia et al. [11] a similar problem is investigated in which MSI contribution to voltage control is ignored. It should be noted that MSI can be the main reactive compensator as a STATCOM as shown in Kayikci and Milanovic [12]. 


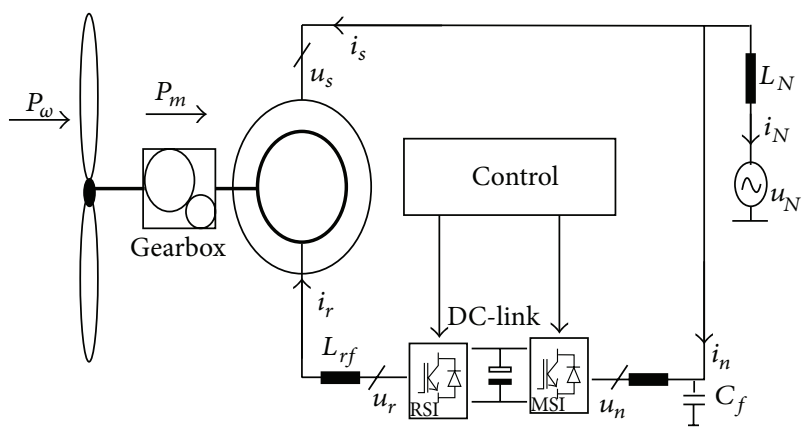

FIGURE 1: DFIG drive topology.

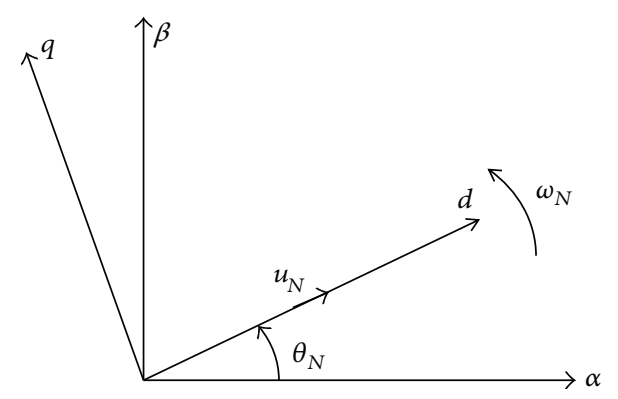

FIGURE 2: Voltage orientation.

An interesting effort has been made on using both the RSI and the MSI to design reactive power regulator in DFIG [13]. There are several other coordinated RSI and MSI based voltage-control methods suggested in the literature (see, e.g., Akhmatov [14] and Ackermann [15] and the references cited therein).

While reactive power control of DFIG has been extensively studied during the past few years, there are some open issues which have practical and theoretical importance in this area. For instance, from a reliable operation and realtime implementation point of view, currently there is no uniform framework for the design of a cost-effective and reliable method for reactive power adjustment. As a matter of fact, in most existing works, either the control development and closed-loop system stability conditions are based on largely oversimplified linear dynamics or the resultant control algorithms are prohibitively too complex for real-time implementation. One reason leading to such barrier is the fact that differential equations of DFIG are nonlinear and complex in nature. To facilitate control design, most existing methods have carried on the tradition of using linear model, without fully recognizing modelling uncertainty, external disturbance, or implementation cost. As such, these control methods which heavily depend on linear model and precise system parameters seldom satisfactorily work in practice. It is interesting to notice that different applications of adaptive control method have been studied in various fields, such as in power systems [16,17] and in robot controlling [18]. Adaptive control method can help to solve the above problems.
This paper proposes a computationally inexpensive control algorithm for controlling reactive power in DFIG for wind turbines. The main interest in the mentioned method is primarily motivated by some practical implementation situations, where algorithm cost-effectiveness appears to be the prior concern. Meanwhile, there exist the possibilities that the system parameters and dynamics are not always fully available for the sake of some constraints. A dynamic model which reflects the electrical connection effects of the MSI of DIFG wind turbine system is established in this paper. Inspired by the recent work on using core information for control design [19], a simple yet effective robust adaptive control scheme is developed. The superior features of the resultant control scheme consist in the significance in dealing with unpredictable lumped disturbances and simple structure. In fact, only little information of the parameters/dynamics is necessary for the construction of the control algorithm. Meanwhile complicated and painful trail-anderror process for control gains determination is no longer needed. These friendly advantages are favourable in practical implementations.

\section{System Topology and Dynamic Equations}

Drive topologies of DFIG have been intensively studied in the literature [13]. Drive topology of DFIG containing the current and power flow is shown in Figure 1. Wind power captured from the wind turbine transfers into electric power through the gear box and the induction generator. The induction generator is quite special since it has a dual converter which is made of electric devices such as IGBTs. The size of the converter is determined according to the desired speed range. With a proper control for the RSI and MSI, the separate control of reactive power from active power is achieved. The DFIG employs some inductors between the rotor terminals and the RSI as filters. In order to suppress harmonics, output filters are also used in the DFIG.

2.1. Voltage Orientation. Considering the deep coupling nature between reactive and active power, a well-chosen orientation can help to control the two variables independently. In this paper, the coordinate system rotates synchronously along with mains voltage vector. By adopting the phaselocked loop (PLL) scheme [20], the mains voltage vector is well tracked by $d$-axis in the frame. Thus we obtain $u_{N}=u_{N d}$ and $u_{N q}=0$. Based on such voltage orientation, current components on the $q$ - and $d$-axis are considered, separately, as reactive component and active component. Thus the $q$-axis current component is responsible for controlling the reactive power production which will be used in later discussion. This implication is illustrated in Figure 2, and $\omega_{N}$ is the angular speed of $u_{N}$.

2.2. Generator Model. In this work, we follow the modelling methods as described in Rabelo et al. [13]. The stator voltage frequency is the same as the net frequency; that is, $\omega_{N}=\omega_{S}$, and the slip frequency is determined by $\omega_{r}=S \omega_{N} \cdot u_{s}$ and 


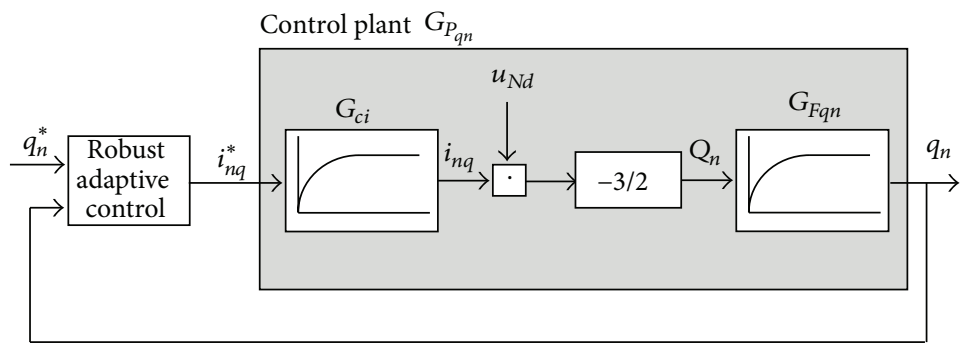

FIGURE 3: Block diagram of the system.

$u_{r}$ are obtained by using the synchronous rotating frame for induction generator in Leonhard [2]. Consider

$$
\begin{aligned}
& u_{r}^{\prime}=R_{r}^{\prime} i_{r}^{\prime}+\frac{d \psi_{r}}{d t}+j S w_{N} \psi_{r}, \\
& u_{s}=R_{s} i_{s}+\frac{d \psi_{s}}{d t}+j w_{N} \psi_{s} .
\end{aligned}
$$

Flux linkage of the rotor $\psi_{r}$ and flux linkage of the stator $\psi_{s}$ are calculated as

$$
\psi_{r}=L_{r}^{\prime} i_{r}^{\prime}+L_{m} i_{s}, \quad \psi_{s}=L_{s} i_{s}+L_{m} i_{r}^{\prime}
$$
by

Electromagnetic torque of the drive system is established

$$
T_{e}=\frac{3}{2} n \frac{L_{m}}{L_{s}} \cdot \mathfrak{J}\left\{\psi_{s} i_{r}^{*}\right\}
$$

where “*” denotes the conjugate complex value and $\mathfrak{\Im}\{\cdot\}$ denotes the imaginary part and $n$ represents pole pairs.

The current components on the stator side with orthogonal coordinate are written as

$$
\begin{gathered}
i_{s d}=-\frac{L_{m}}{L_{s}} i_{r d}, \\
i_{s q}=\frac{\psi_{s q}}{L_{s}}-\frac{L_{m}}{L_{s}} i_{r q},
\end{gathered}
$$

where $i_{s d}$ is the stator current component on the $d$-axis and $i_{s q}$ is the stator current component on the $q$-axis.

Using the flux linkage equations above and replacing the stator current component, the following equations can be obtained:

$$
\begin{gathered}
u_{r q}=R_{r} i_{r q}+\sigma L_{r} \frac{d i_{r q}}{d t}-S w_{N} \sigma L_{r} i_{r q} \\
u_{r d}=R_{r} i_{r d}+\sigma L_{r} \frac{d i_{r d}}{d t}+S w_{N} \sigma L_{r} i_{r q}+S w_{N} \frac{L_{m}}{L_{s}} \psi_{s q}
\end{gathered}
$$

where $\sigma=1-\left(\mathrm{Lm}^{2} / L_{s} L_{r}\right)$. The basic electrical formulas above will be used to construct the inner rotor current controller.
2.3. LC Filter Model. Considering the inverter synchronized with the mains voltage and ignoring the voltage drop on the $L_{N}$, the MSI output current dynamics can be described as follows:

$$
\begin{gathered}
u_{N q}=R_{f} i_{n q}+L_{f} \frac{d i_{n q}}{d t}-w_{N} L_{f} i_{n d}+u_{n q}=0, \\
u_{N d}=R_{f} i_{n d}+L_{f} \frac{d i_{n d}}{d t}-w_{N} L_{f} i_{n q}+u_{n d} .
\end{gathered}
$$

The block diagram in Figure 1, together with the above equations, indicated the mutual and internal relationship in the drive topology of DFIG and the wind power generation system.

\section{Reactive Power Control Design}

Considering the external disturbance acting on the system, reactive power at the MSI is governed by the dynamic equation as given in (8): that is,

$$
\ddot{q}_{n} T_{1} T_{2}+\dot{q}_{n}\left(T_{1}+T_{2}\right)+q_{n}+h(\cdot)=K_{q n} i_{n q}^{*},
$$

which can be shown in detail as follows.

3.1. Current Inner Loop Control. This is a cross-coupled 2D problem. The transfer function of the plant $G_{i}(s)$ is described as

$$
G_{i}(s)=\frac{K_{i}}{s T_{i}+1},
$$

where $K_{i}=1 / R$ and $T_{i}=L / R$. The transfer functions are identical on both $d$ and $q$ axes. The time delay of signal preconditioning and processing can be regarded as a small time constant $T_{\text {sum }} \ll T_{i}$. Note that $T_{\text {sum }}$ is small and its accurate value is normally difficult to obtain. The first-order transfer function of such time constant part is described as

$$
G_{\text {sum }}(s)=\frac{1}{s T_{\text {sum }}+1} .
$$

By utilizing of a simple controller $G_{R i}$, the following openloop transfer function is established:

$$
G_{o i}(s)=G_{R i} G_{i} G_{\text {sum }}=K_{P i} \frac{s T_{I i}+1}{s T_{I i}} \frac{K_{i}}{s T_{i}+1} \frac{1}{s T_{\text {sum }}+1},
$$

where $K_{P i}$ is the differential parameter and $T_{I i}$ is the integrated time. 
3.2. Output Current Control. Output current control should track the input current without heavy overshoots. A general method to solve such problems is proposed in Föllinger [21]. To achieve a well damping factor $1 / \sqrt{2}$, we can set $K_{P i}$ and $T_{I i}$ as follows:

$$
K_{P i}=\frac{L}{2 T_{\text {sum }}}, \quad T_{I i}=T_{i} .
$$

The closed-loop transfer function becomes

$$
G_{c i}(s)=\frac{G_{o i}}{1+G_{o i}}=\frac{1}{2 T_{\mathrm{sum}}^{2} s^{2}+2 T_{\mathrm{sum}} s+1} .
$$

Since $T_{\text {sum }} \ll T_{i}$, the square value in the $s^{2}$ term can be ignored. Thus, the closed-loop function of the current inner control is simplified and obtained as

$$
G_{c i}(s) \cong \frac{1}{2 T_{\text {sum }} s+1} .
$$

At the mains-side inverter and under the voltage orientation discussed above, the reactive power is established by

$$
Q_{n}=\frac{3}{2} \Im\left\{u_{n} i_{n}^{*}\right\}=\frac{3}{2}\left(u_{N q} i_{n d}-u_{N d} i_{n q}\right)=-\frac{3}{2} u_{N d} i_{n q} .
$$

Before passing into the controller, the data of the input reactive power pass through a filter. The filter is given by a one-order transfer function as described in Rabelo et al. [13]. Consider

$$
G_{F_{q n}}=\frac{q_{n}}{Q_{n}}=\frac{1}{\left(s T_{F_{q n}}+1\right)},
$$

where $q_{n}$ is the ultimate actual reactive power through the filter and $T_{F_{q n}}$ is the filter time constant.

We take the current inner control in (13) into account. Thus the control plant becomes the following second-order transfer function:

$$
G_{P_{q n}}=K_{q n} G_{c i} G_{F_{q n}}=\frac{K_{q n}}{\left(s 2 T_{\text {sum }}+1\right)\left(s T_{F_{q n}}+1\right)},
$$

where

$$
K_{q n}=-\left(\frac{3}{2}\right) u_{N d}
$$

The block diagram of the system adopting robust adaptive control methods is shown in Figure 3. The primary objective is to build the reference current $i_{n q}^{*}$ which makes the actual reactive power $q_{n}$ regulate the reference one $q_{n}^{*}$ asymptotically.

We rewrite control plant equation (16) as

$$
\frac{q_{n}}{i_{n q}^{*}}=\frac{K_{n q}}{\left(s T_{1}+1\right)\left(s T_{2}+1\right)},
$$

where $T_{1}=2 T_{\text {sum }}, T_{2}=T_{F_{n q}}$. Using Laplace inverse transform, it follows that

$$
\ddot{q}_{n} T_{1} T_{2}+\dot{q}_{n}\left(T_{1}+T_{2}\right)+q_{n}=K_{q n} i_{n q}^{*} .
$$

Taking the external disturbances acting on the system into account, (20) becomes (8) where $h(\cdot)$ is the external disturbances.
3.3. Robust Adaptive Control for Mains-Side Reactive Power. In this section, a robust adaptive control for mains-side reactive power will be proposed. To build a meaningful adaptive control scheme, two realistic assumptions are adopted.

Assumption 1. Voltage amplitude at net connecting point remains nonzero. Thus with the voltage orientation and (6), $u_{N d}$ can be regarded as a positive known number so that $K_{q n}$ with respect to (18) is a negative known number.

Remark 2. Assumption 1 imposed here, rather standard in addressing system stabilization, is practical because the wind power generation system will be shut off if the voltage at the net is extremely low; thus the zero voltage does not occur for the situation under consideration.

To design the tracking controller, we define the reactive power tracking error as

$$
e=q_{n}-q_{n}^{*}
$$

To simplify controller design, we introduce $\varepsilon$ and define $\varepsilon$ as

$$
\varepsilon=\beta e+\dot{e},
$$

where $\beta>0$ is a designed constant.

Apparently if $\varepsilon$ converges to zero as time increases, $e$ and $\dot{e}$ also converge to zero, which means if we can design a controller that forces $\varepsilon$ to converge to zero as time increases, then problem will be solved.

Taking derivative of (22) with respect to time, we get

$$
\dot{\varepsilon}=\beta \dot{e}+\ddot{e} .
$$

Substituting $\ddot{e}$ with (21) and using $\ddot{q}_{n}$ as given in (8), we obtain

$$
\dot{\varepsilon}=\frac{K_{q n}}{T_{1} T_{2}} i_{n q}^{*}+\frac{1}{T_{1} T_{2}}\left[-h(\cdot)-q_{n}-\left(T_{1}+T_{2}\right) \dot{q}_{n}\right]+\beta \dot{e}-\ddot{q}_{n}^{*} .
$$

Or equivalently,

$$
\dot{\varepsilon}=B u+L(\cdot),
$$

where $u=-i_{n q}^{*}$,

$$
\begin{gathered}
B=-\frac{K_{q n}}{T_{1} T_{2}}, \\
L(\cdot)=\frac{1}{T_{1} T_{2}}\left[-h(\cdot)-q_{n}-\left(T_{1}+T_{2}\right) \dot{q}_{n}\right]+\beta \dot{e}-\ddot{q}_{n}^{*} .
\end{gathered}
$$

Obviously $B$ is positive because of the definition of $K_{q n}$ as given in (18). Note that

$$
L(\cdot) \leq\left|\frac{1}{T_{1} T_{2}}\right|\left[|h(\cdot)|+\left|q_{n}\right|+\left|\left(T_{1}+T_{2}\right)\right|\left|\dot{q}_{n}\right|\right]+|\beta \dot{e}|+\left|\ddot{q}_{n}^{*}\right| .
$$


Assumption 3. In this assumption, the characteristic of the disturbance is discussed. The external disturbance $h(\cdot)$ is bounded which leads to the existence of an unknown constant such that

$$
|h(\cdot)| \leq a_{1}<\infty
$$

Also, the time constants $T_{1}$ and $T_{2}$, although unknown in general, are bounded so that

$$
\left|\frac{1}{T_{1} T_{2}}\right| \leq a_{2}<\infty, \quad\left|\left(T_{1}+T_{2}\right)\right| \leq a_{3}<\infty,
$$

where $a_{2}$ and $a_{3}$ are some unknown positive constants.

This assumption, quite reasonable in practice, allows for the establishment of

$$
|L(\cdot)| \leq a \varphi\left(q_{n}\right)
$$

where

$$
\begin{aligned}
a & =\max \left(a_{2} \cdot a_{1}, a_{2}, a_{2} \cdot a_{3}, 1\right), \\
\varphi\left(q_{n}\right) & =1+\left|q_{n}\right|+\left|\dot{q}_{n}\right|+\beta|\dot{e}|+\left|\ddot{q}_{n}^{*}\right| .
\end{aligned}
$$

We design the input as

$$
u=-\left(K_{0}+\widehat{K}\right) \varepsilon
$$

where $K_{0}>0$ is a design constant and $\widehat{K}$ is updated as

$$
\widehat{K}=\frac{\widehat{a} \varphi}{|\varepsilon|},
$$

where $\widehat{a}$ is the estimation of $a$ and is updated as

$$
\dot{\hat{a}}=|\varepsilon| \varphi .
$$

Theorem 4. For such system established by (8) under the assumptions, if $u$ is calculated by (33a), (33b), and (33c), then the reactive power is ensured to track the desired one asymptotically.

Proof. The result can be justified using the following Lyapunov function:

$$
V=\frac{1}{2} \varepsilon^{2}+\frac{1}{2 B_{\min }}\left(a-\hat{a} B_{\min }\right)^{2},
$$

where $B_{\text {min }}$ is constant and $0<B_{\text {min }} \leq B$ for $\forall B \in L_{\infty}$.

Differentiating $V$ leads to

$$
\dot{V}=\varepsilon \dot{\varepsilon}-\left(a-\widehat{a} B_{\min }\right) \dot{\hat{a}} .
$$

From (25) and (33a), we get

$$
\dot{\varepsilon}=-B K_{0} \varepsilon-B \widehat{K} \varepsilon+L
$$

Equation (35) becomes

$$
\dot{V}=\varepsilon\left(-B K_{0} \varepsilon-B \widehat{K} \varepsilon+L\right)-\left(a-\widehat{a} B_{\min }\right) \dot{\hat{a}} .
$$

Substituting $\widehat{K}$ with (33b) and $\dot{\hat{a}}$ with (33c),

$$
\begin{aligned}
\dot{V} & =\varepsilon L-B K_{0} \varepsilon^{2}-B \widehat{a} \varphi|\varepsilon|-\varphi|\varepsilon|\left(a-\widehat{a} B_{\text {min }}\right) \\
& \leq|\varepsilon||L|-B K_{0} \varepsilon^{2}-B \widehat{a} \varphi|\varepsilon|-\varphi|\varepsilon|\left(a-\widehat{a} B_{\min }\right) \\
& =-B K_{0} \varepsilon^{2}+(|\varepsilon||L|-|\varepsilon| \varphi a)+\left(B_{\min } \widehat{a} \varphi|\varepsilon|-B \widehat{a} \varphi|\varepsilon|\right) .
\end{aligned}
$$

Because we have

$$
|L(\cdot)| \leq a \varphi, \quad 0<B_{\min } \leq B,
$$

we can get

$$
\dot{V} \leq-B_{\min } K_{0} \varepsilon^{2}<0 .
$$

As $B_{\min }>0$, it is readily shown from (40) that both $\varepsilon$ and $\widehat{a}$ are bounded. Furthermore, we can show that $\dot{\varepsilon}$ is bounded and thus $\varepsilon$ is uniformly continuous. Therefore, using Barbalat Lemma [22] the reality is obtained that $\varepsilon$ converges to zero asymptotically. By utilizing (22), ultimately, $e$ and $\dot{e}$ converge to zero asymptotically; then the result is established.

Remark 5. It should be noted that when the states get closer to zero, the control scheme might experience chattering. This, however, can be avoided by using the simple but classic means of replacing $z /|z|$ with $z /(|z|+\tau)$, where $\tau$ is small. Meanwhile, in order to prevent the estimate $\widehat{a}$ from drifting, (33c) can be modified to

$$
\dot{\hat{a}}=-\sigma_{1} \widehat{a}+\sigma_{2} \frac{\varepsilon^{2} \varphi^{2}}{|\varepsilon| \varphi+\tau}, \quad \sigma_{1}>0, \sigma_{2}>0 .
$$

In this case, we have the following ultimately uniformly bounded (UUB) tracking control result.

Theorem 6. Also for system established by (8) under the assumptions, if the following robust adaptive control algorithm is adopted,

$$
u=-\left(K_{0}+\widehat{K}\right) \varepsilon,
$$

where $K_{0}>0$ is a design constant and $\widehat{K}$ is updated as

$$
\widehat{K}=\frac{\widehat{a} \varphi}{|\varepsilon|+\tau}
$$

where $\hat{a}$ is the estimation of $a$ and is updated by (33a), then the system is ensured to be UUB stable.

Proof. This theorem can be proved by utilizing of the methods in Cai et al. [23].

Remark 7. Instead of using PI controller as in Rabelo et al. [13], a simple robust adaptive control scheme is developed here in which one only needs to specify the parameters in a clear direction; that is, $K_{0}>0$ and $\beta>0$.

Remark 8. The significance of the developed control scheme is twofold. 
(1) The control scheme developed here does not rely on the precise value for the time constants $T_{1}, T_{2}$. Also, there is no need for analytical estimation of the unknown parameters $a_{1}, a_{2}, a_{3}$. Such fact can sufficiently simplify the design procedure and implementation of the proposed control algorithm.

(2) As the parameter $\hat{a}$ involved in the controller is updated automatically via the algorithm, and such process is independent of operation conditions, no redesign or reprogramming is needed during the system operation.

\section{Simulation Verification}

Aiming at validating the correctness of the robust adaptive reactive control scheme, simulations with Matlab/Simulink are presented here.

Per unit (p.u.) value is introduced to simplify calculation and simulation. The datum voltage $V_{a v}$ and datum capacity $S_{a v}$, respectively, are set as $330 \mathrm{~V}$ and $1 \mathrm{MVar}$. The net voltage $u_{N d}$ under voltage orientation is chosen as $220 \mathrm{~V}$ and thus $K_{q n}$ with respect to (17) is calculated as -1 . Other parameters used for simulation are chosen as $T_{\text {sum }}=0.5, T_{F_{q n}}=1, \beta=1$, and $K_{0}=1$. The PI control algorithm described in Ackermann [15] for reactive power is rebuilt for comparison. Three types of working conditions are simulated here.

4.1. Regulating under Steady Working Condition. In real application, DFIG can work as a compensator to provide constant reactive power. Based on this fact, we set the desired reactive power output as 1 p.u. The desired reactive power and actual one are together plotted in Figure 4.

Compared with the adopted PI scheme, adaptive method eliminates overshoot and has a shorter regulate time and a longer rise time. Both of the control schemes can obtain stabilization.

4.2. Regulating under Modelling Uncertainty. In this kind of simulation, the influence of modelling uncertainty is investigated. Assume that the time constant $T_{\text {sum }}$ has a deviation of $5 \%$; then $T_{\text {sum }}=0.525$. The result of the proposed adaptive method is illustrated in Figure 5 and the PI method is shown in Figure 6.

The result shows that, by utilizing the proposed scheme, the tracking trajectory almost remains the same even under such modelling uncertainty and parameter deviation. From the point of detail, the proposed adaptive scheme enjoys a better tracking trajectory compared with the PI controller under such condition.

4.3. Tracking under Dynamic Reactive Power Compensation. If the voltage begins to drop during an unsymmetrical grid fault, the DFIG will work as a dynamic reactive compensator to keep the voltage to a certain degree. Once the fault is moved, the reactive power generating capacity of DFIG will be resumed [24]. Based on this fact, we set the desired reactive power output as illustrated in Figure 7. Specifically, the DFIG is ordered to provide 1 p.u. before $7 \mathrm{~s}$ and provide an extra

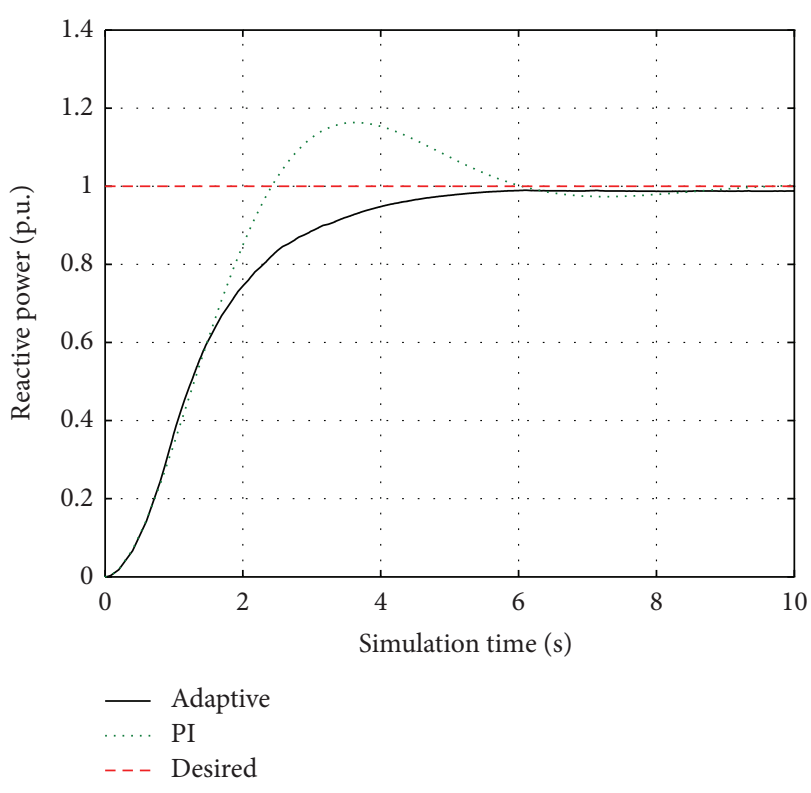

FIgURE 4: Reactive power regulation trajectories.

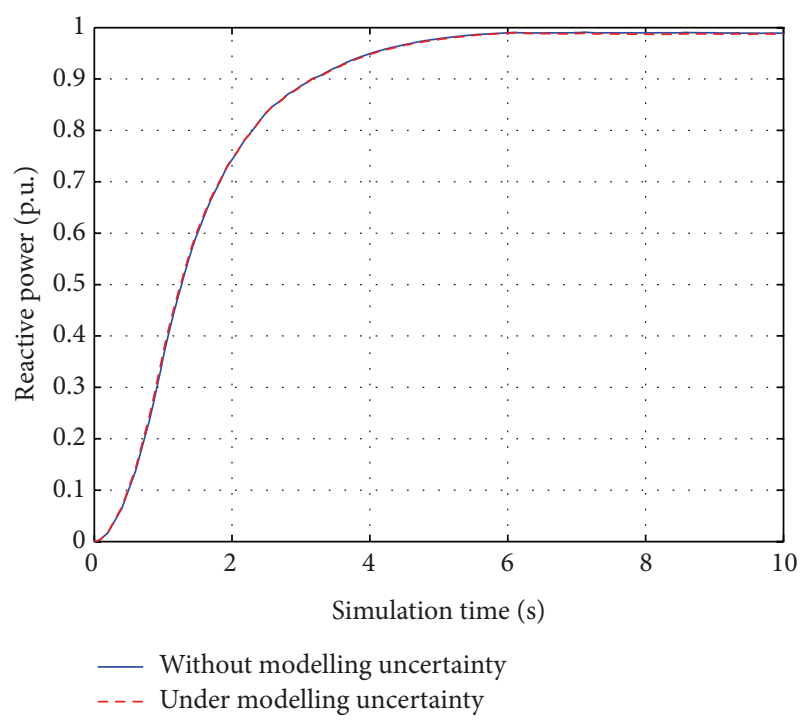

FIGURE 5: Adaptive controller tracking trajectories.

0.2 p.u. reactive power in the time from $7 \mathrm{~s}$ to $8 \mathrm{~s}$, because the proposed scheme uses the derivative of the desired input. The reference has been smoothed before putting into the controller.

The result of PI controller is also compared to the proposed adaptive method. Figure 7 illustrates that, compared with PI controller, the proposed method can track the desired reactive power better. This is because PI controller fails to track the reference value at the time of $7 \mathrm{~s}$. The result shows that the adopted PI controller fails to deal with such problem which, however, the adaptive scheme proposed in this paper can deal with. 


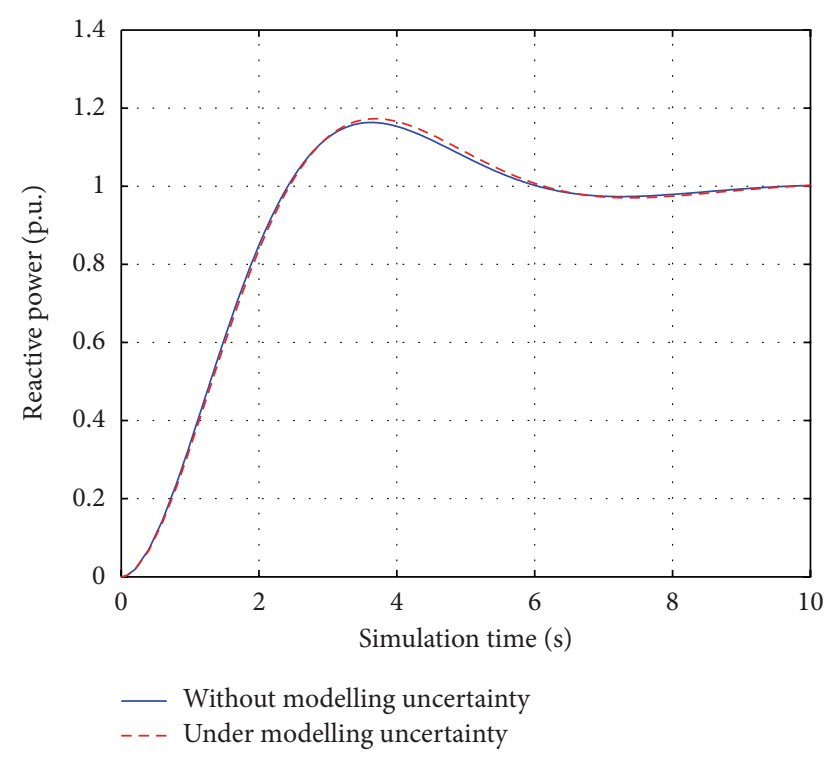

FIGURE 6: PI controller tracking trajectories.

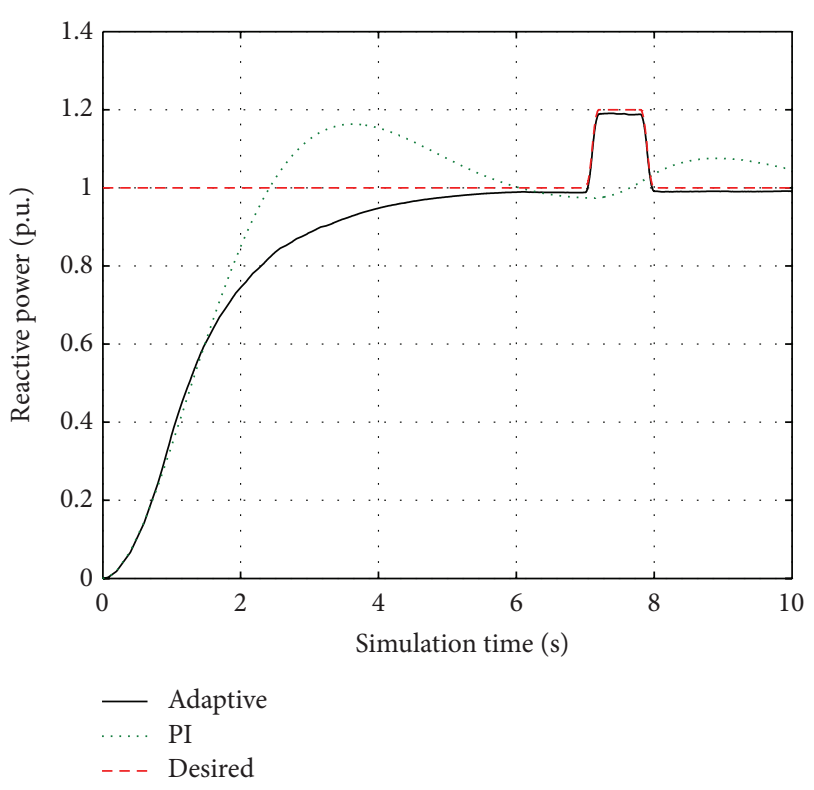

FIGURE 7: Reactive power tracking trajectories.

\section{Conclusions}

Reactive power control for mains-side inverter (MSI) in DFIG represents an important issue in wind power generation systems. A robust adaptive control scheme for MSI is developed. As confirmed by theoretical analysis the proposed method is able to maintain satisfactory performance under varying operation conditions without the need for manually redesigning or reprogramming the control gains. Numerical simulations also validate the correctness and benefits of the proposed algorithm.

\section{Conflict of Interests}

The authors declare that there is no conflict of interests regarding the publication of this paper.

\section{Acknowledgments}

This work was supported in part by the National Basic Research Program of China (973 Program no. 2012CB215200) and in part by the National Natural Science Foundation of China (no. 51205046).

\section{References}

[1] Y. She, X. She, and M. E. Baran, "Universal tracking control of wind conversion system for purpose of maximum power acquisition under hierarchical control structure," IEEE Transactions on Energy Conversion, vol. 26, no. 3, pp. 766-775, 2011.

[2] W. Leonhard, Control of Electrical Drives, Springer, 2000.

[3] Rabelo and B. Hofmann W, "Power flow optimisation and grid integration of wind turbines with the Doubly-Fed induction generator," in Proceedings of the 36th International Conference on Power Electronics Specialists Conference, pp. 2930-2936, Recife, Brazil, June 2005.

[4] R. Pena, J. C. Clare, and G. M. Asher, "Doubly fed induction generator using back-to-back PWM converters and its application to variable-speed wind-energy generation," IEE Proceedings: Electric Power Applications, vol. 143, no. 3, pp. 231241, 1996.

[5] M. El Moursi, G. Joos, and C. Abbey, "A secondary voltage control strategy for transmission level interconnection of wind generation," IEEE Transactions on Power Electronics, vol. 23, no. 3, pp. 1178-1190, 2008.

[6] E. O. N. Netz, “Grid code high and extra high voltage," 2006.

[7] T. K. A. Brekken and N. Mohan, "Control of a doubly fed induction wind generator under unbalanced grid voltage conditions," IEEE Transactions on Energy Conversion, vol. 22, no. 1, pp. 129135, 2007.

[8] M. Machmoum, F. Poitiers, C. Darengosse, and A. Queric, "Dynamic performances of a doubly-fed induction machine for a variable-speed wind energy generation," in Proceedings of the International Conference on Power System Technology, pp. 24312436, October 2002.

[9] S. Engelhardt, I. Erlich, C. Feltes, J. Kretschmann, and F. Shewarega, "Reactive power capability of wind turbines based on doubly fed induction generators," IEEE Transactions on Energy Conversion, vol. 26, no. 1, pp. 364-372, 2011.

[10] J. G. Slootweg, S. W. H. de Haan, H. Polinder, and W. L. Kling, "Voltage control methods with grid connected wind turbines: a tutorial review," Wind Engineering, vol. 25, no. 6, pp. 353-366, 2001.

[11] A. Tapia, G. Tapia, J. Xabier Ostolaza, and J. R. Sáenz, "Modeling and control of a wind turbine driven doubly fed induction generator," IEEE Transactions on Energy Conversion, vol. 18, no. 2, pp. 194-204, 2003.

[12] M. Kayikci and J. V. Milanovic, "Reactive power control strategies for DFIG-based plants," IEEE Transactions on Energy Conversion, vol. 22, no. 2, pp. 389-396, 2007.

[13] B. C. Rabelo Jr., W. Hofmann, J. L. da Silva, R. G. de Oliveira, and S. R. Silva, "Reactive power control design in doubly fed 
induction generators for wind turbines," IEEE Transactions on Industrial Electronics, vol. 56, no. 10, pp. 4154-4162, 2009.

[14] V. Akhmatov, Analysis of Dynamic Behaviour of Electric Power Systems with Large Amount of Wind Power, Electric Power Engineering, Ørsted-DTU, Technical University of Denmark, 2003.

[15] T. Ackermann, Ed., Wind Power in Power Systems, John Wiley and Sons, New York, NY, USA, 2005.

[16] Y. Song, X. Li, and W. Cai, "Adaptive and fault-tolerant reactive power compensation in power systems," International Journal of Innovative Computing, Information and Control, vol. 9, no. 8, pp. 3403-3413, 2013.

[17] D. Niu and Y. Wei, "A novel social-environmental-economic dispatch model for thermal/wind power generation and application," International Journal of Innovative Computing, Information and Control, vol. 9, no. 7, pp. 3005-3014, 2013.

[18] Y. Chen, G. Mei, G. Ma, S. Lin, and J. Gao, "Robust adaptive inverse dynamics control for uncertain robot manipulator," International Journal of Innovative Computing, Information and Control, vol. 10, no. 2, pp. 575-587, 2014.

[19] Y.-D. Song, H.-N. Chen, and D.-Y. Li, "Virtual-point-based fault-tolerant lateral and longitudinal control of $4 \mathrm{~W}$-steering vehicles," IEEE Transactions on Intelligent Transportation Systems, vol. 12, no. 4, pp. 1343-1351, 2011.

[20] V. Kaura and V. Blasko, "Operation of a phase locked loop system under distorted utility conditions," IEEE Transactions on Industry Applications, vol. 33, no. 1, pp. 58-63, 1997.

[21] O. Föllinger, Regelungstechnik, Springer, 8th edition, 1999.

[22] J. J. Slotine and W. Lei, Applied Nonlinear Control, Prentice-Hall, 1991.

[23] W. Cai, X. H. Liao, and Y. D. Song, "Indirect robust adaptive fault-tolerant control for attitude tracking of spacecraft," Journal of Guidance, Control, and Dynamics, vol. 31, no. 5, pp. 1456-1463, 2008.

[24] S. Seman, J. Niiranen, and A. Arkkio, "Ride-through analysis of doubly fed induction wind-power generator under unsymmetrical network disturbance," IEEE Transactions on Power Systems, vol. 21, no. 4, pp. 1782-1789, 2006. 


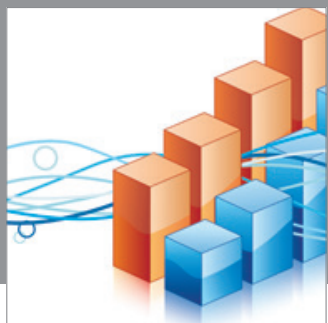

Advances in

Operations Research

mansans

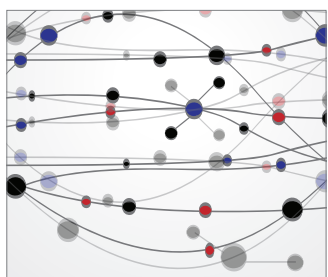

The Scientific World Journal
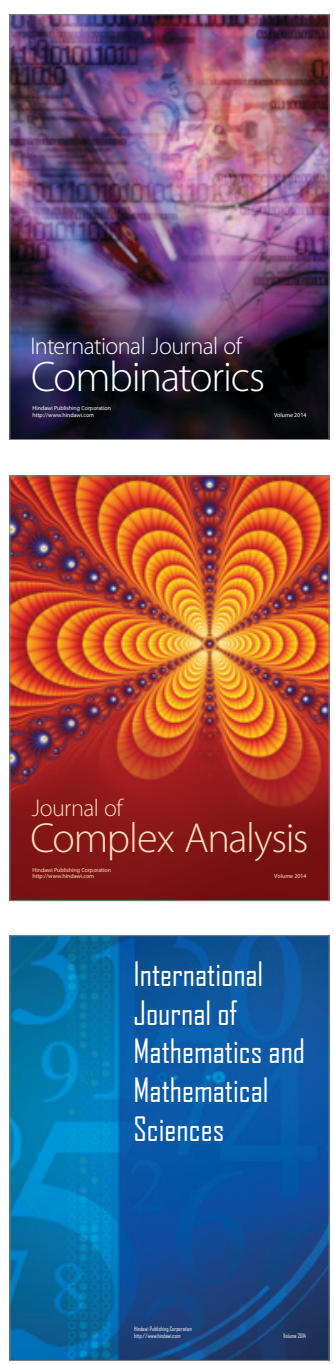
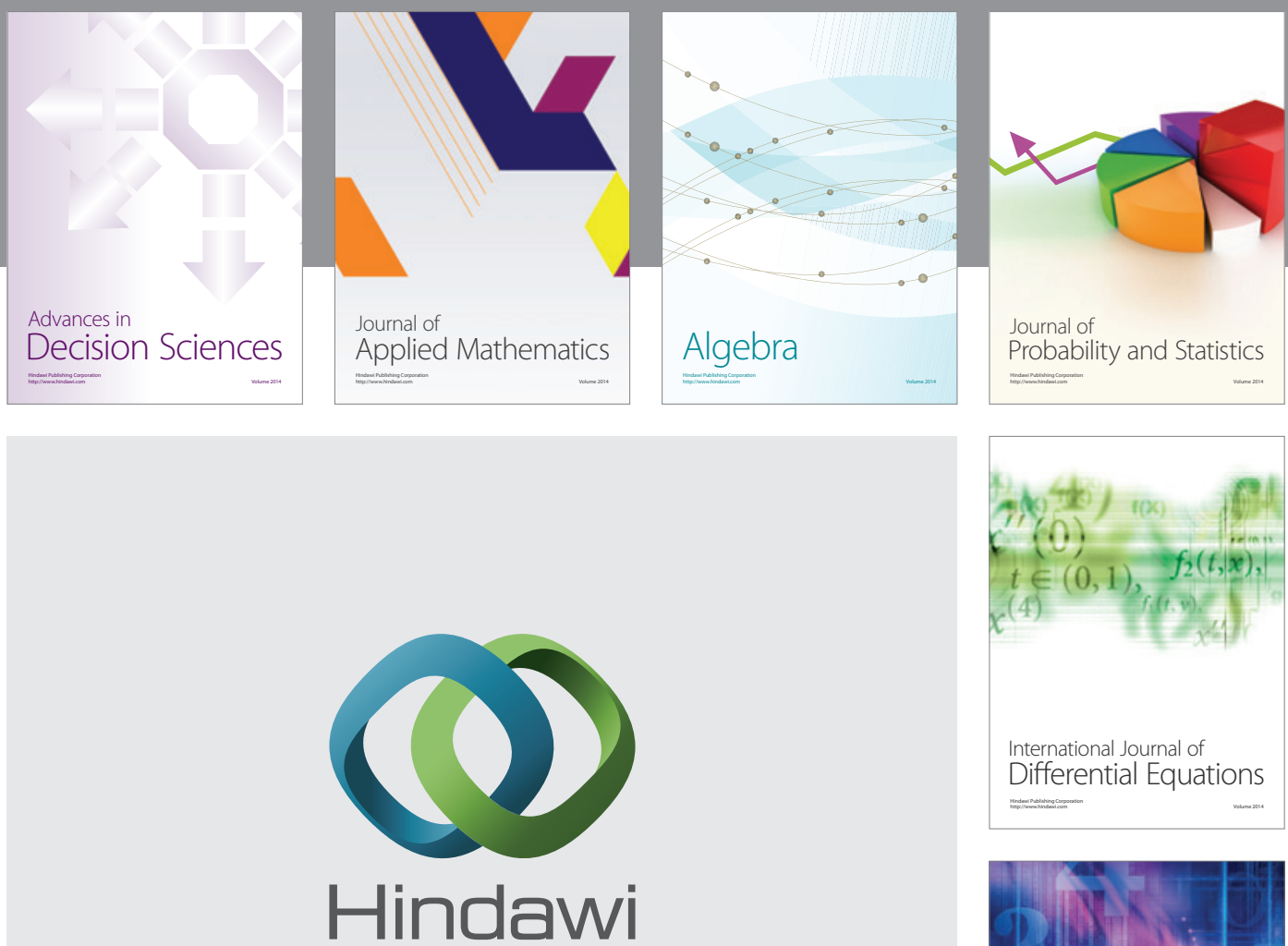

Submit your manuscripts at http://www.hindawi.com
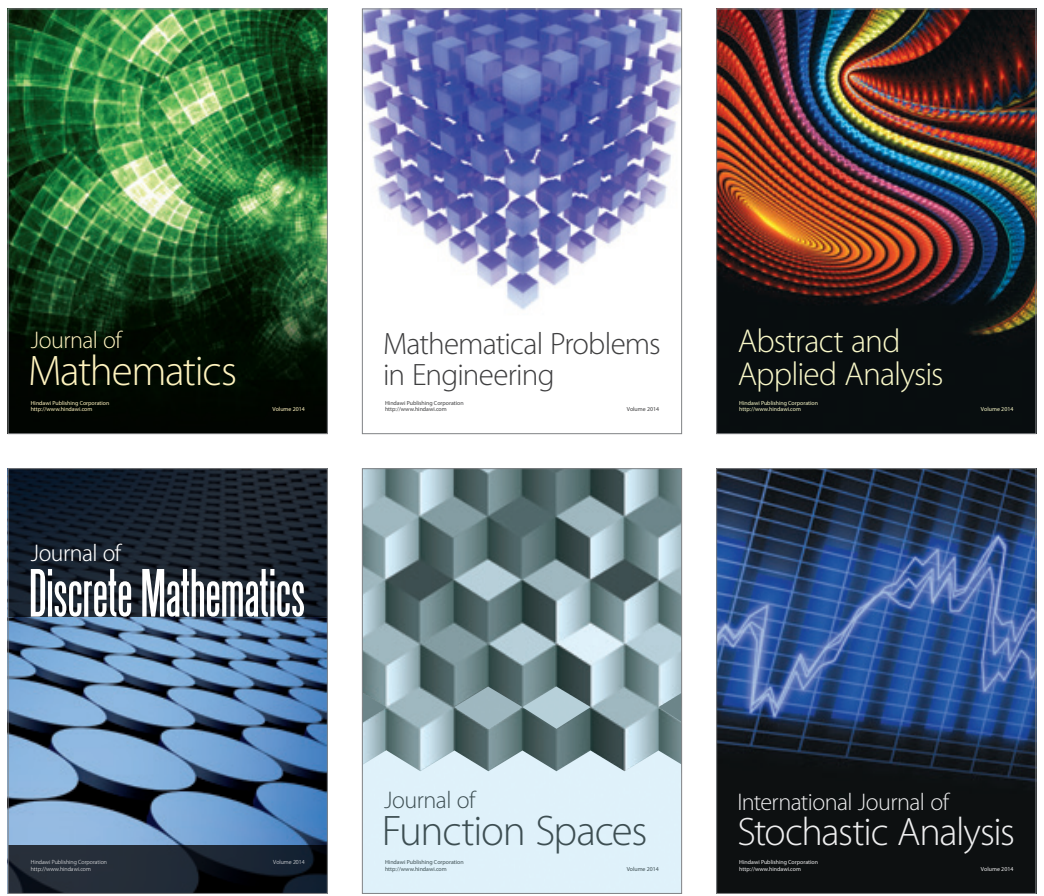

Journal of

Function Spaces

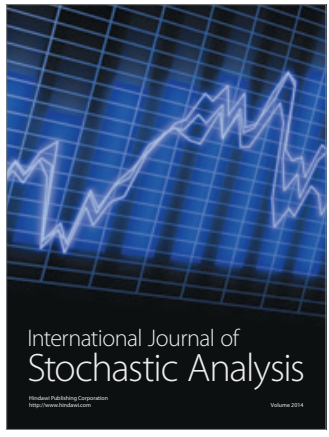

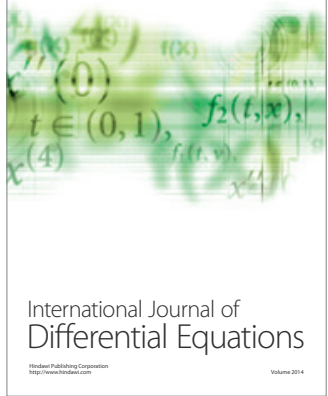
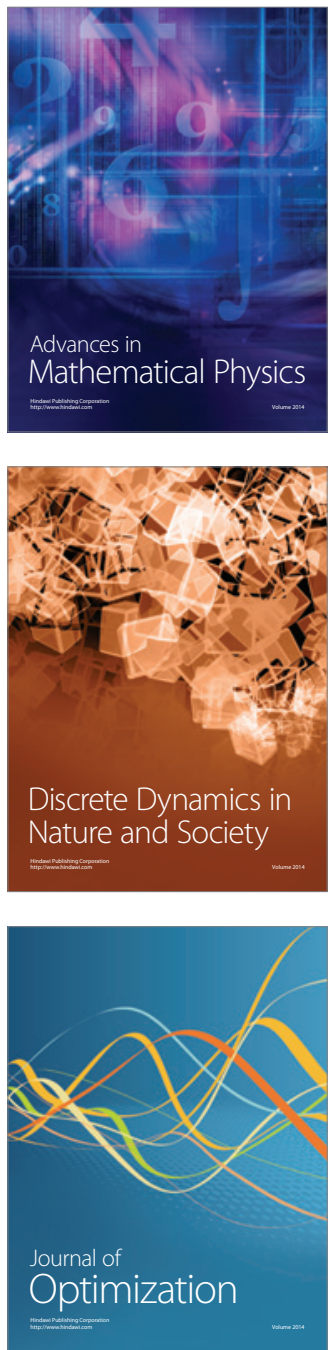